\title{
Effect of Exercise Program on Acromiohumeral Distance in Subacromial Impingement Syndrome
}

\section{Lilian Albert Zaky', Nagy Ahmed Zaki Mostafa Sabet², Maha Mostafa Mohamed ${ }^{3}$, Ahmed Elhamy Mosad ${ }^{4}$}

${ }^{1}$ Assistant Professor in the Department of Physical Therapy for Musculoskeletal Disorders and its Surgery, Faculty of Physical Therapy, Cairo University, Egypt

${ }^{2}$ Professor of Orthopaedic Surgery Faculty of Medicine Misr University for Science and Technology ${ }^{3}$ Lecturer in the Department of Physical Therapy for Musculoskeletal Disorders and its Surgery, Faculty of Physical Therapy, Cairo University, Egypt

${ }^{4}$ Demonstrator in the Department of Physical Therapy for Musculoskeletal Disorders and its Surgery, Faculty of Physical Therapy, MISR University for Science and Technology, Egypt

\section{ABSTRACT}

Among painful shoulder conditions, Shoulder Subacromial impingement syndrome is the most common cause, Acromiohumeral distance changes commonly reported in shoulder impingements.

Objective: of this study was to evaluate the association between Acromio Humeral distance (AHD) changes and pain and upper limb function before and after physiotherapy rehabilitation program in patients with Subacromial impingement (SIS) using ultrasonographic imaging.

Methods: Fifteen patients suffered from shoulder impingement were recruited for this study. Their age ranged from 25-45 years. Acromiohumeral distance was examined by using the Ultrasonography at three abduction angles $(0,45,60)$ Pain level was assessed by Visual analog scale and Upper extremity function was assessed using Disability of the arm shoulder and hand questioner.

Patients were assessed before the study and an exercise rehabilitation program that lasted for four weeks then reassessed again.

Design: prospective single group pre-post design

Results: showed significant changes in all measured variables as a result of the therapeutic exercise program. The results proved that Exercise not only improved AcromioHumeral distance at all abduction angles.

Conclusion: Therapeutic exercise programs have a positive effect on the size of the Subacromial space confirming the external mechanism of shoulder impingement.

Keywords: Shoulder Subacromial impingement, Ultrasonography, Acromiohumeral distance, Therapeutic exercises, Shoulder imaging. 


\section{INTRODUCTION}

Shoulder complaints considered the most commonly encountered musculoskeletal complained in primary clinical practice after neck and back pain ${ }^{[1]}$. The condition is commonly termed as Subacromial shoulder impingement (SIS), which characterized by narrowing of the Subacromial space that Leeds to an encroachment of the Subacromial soft tissue structures underneath ${ }^{[2]}$.

The etiology SIS is multifactorial as anatomical, biomechanical, as well as psychosocial factors, are likely involved ${ }^{[1]}$. While the etiology, paingenerating mechanisms, and level of soft tissue involvement of rotator cuff is debatable, there is a growing body of evidence that suggests that the Acromio Humeral distance (AHD) is associated with the presence and magnitude of rotator cuff tears ${ }^{[3]}$.

The Acromio Humeral distance (AHD) of the Subacromial space and its dynamic changes when the arm is elevated, is believed to be involved in the etiology of SIS and it has been proposed that its normalization might be involved in the positive rehabilitation outcome ${ }^{[4]}$.

Acromio Humeral distance changes have been found in healthy subjects when compared to SIS subjects using magnetic resonance imaging (MRI) or a simple X- ray technique ${ }^{[5]}$. Recently, ultrasonography (US) has been increasingly used to study and evaluate the SIS patients. US is cheaper than MRI, radiation-free, and considered costeffective clinical tool to measure SIS at the outlet via the AHD ${ }^{[6]}$.

Ultrasound imaging of AHD is obtained with the linear transducer oriented placed on the lateral aspect of the acromion to capture both the acromion and the superior portion of the humeral head and there are studies that proven the validity and reliability of these techniques ${ }^{[7]}$.

Systematic reviews of Physical therapy for rotator cuff pathology and shoulder impingement suggest that therapeutic exercises may be an effective treatment ${ }^{[8]}$. Studies also strongly suggest that exercise improves symptoms in patients with impingement syndrome and the effect of exercise may be augmented with manual therapy ${ }^{[9]}$.

\section{SUBJECTS AND METHODS}

Fifteen subjects from both sexesmedically diagnosed with SIS. They were referred to physical therapy from the orthopedic outpatient clinic of the faculty of medicine, Misr University for science and technology, Egypt. All subjects were informed prior to the participation of the study and signed a consent form.

Subjects included in the study groups their mean age was 32.46 SD 6.03 years old and the duration of illness mean was 6.51 SD 2.77. Exclusion criteria were shoulder instabilities, history of fractures or surgeries in the involved upper limb, cervical pathology radiating to the affected arm, rheumatic diseases, acromial shape abnormalities, adhesive causalities. calcified tendonitis, osteoporosis, and instability.

\section{INSTRUMENTATION AND MATERIALS}

Ultrasonic imaging measurement of the AHD is obtained by holding the transducer longitudinally at the outlet of the Subacromial space. The transducer then slightly moves around to find the smallest AHD at the prober abduction angle, which then measured and recorded in millimeters. This technique proved to be reliable ${ }^{[10]}$.

The universal visual analogue scale (VAS) for perceived pain level assessment used in the study. The VAS tested for its reliability by and found to be reliable ${ }^{[11]}$. Functional assessment performed using DASH questioner ${ }^{[12]}$

\section{STUDY PROTOCOL}

Each patient was treated for twelve sessions three days per week for a total period of four weeks at the out-patient clinic of the faculty of Physical Therapy, Misr University for Science and Technology.

The selected patients had their pain level assessment using visual analogue scale (VAS) and functional disability level of the upper extremity using disability of the arm, shoulder and hand (DASH) index and sonographic assessment of the AHD 
using mindray machine (mindray DP - 2200) at 0 , 45 and 60 shoulder abduction before and after the exercise rehabilitation program based on the guidelines from $^{[13]}$.

Exercise rehabilitation program included patient's education and advice, postural exercises, ice application, stretching exercises, a range of motion exercise, strengthening exercises and scapular stabilization exercises.

\section{ETHICAL CONSIDERATIONS}

All patients were informed of the purpose, tools, procedures, and duration of the study and signed a written consent

\section{STATISTICAL ANALYSIS}

Statistical analysis was conducted using SPSS for windows, version 18 (SPSS, Inc., Chicago, IL). The current test involved two independent variables. The first one was the (tested group); between subject factor which had one level (study group). The second one was the (measuring periods); within subject factor which had two levels (pre and post treatment). In addition, this test involved five tested dependent variables (AHD at 0, AHD at 45, AHD at 60 angles, pain level, and DASH). Accordingly, "One way within subject MANOVA" was used to compare between "pre" and "post treatment" tests for each dependent variables for the study group with the alpha level 0.05 .

Prior to final analysis, data were screened for normality assumption, and presence of extreme scores. This exploration was done as a pre-requisite for parametric calculations of the analysis of difference.

Descriptive analysis using histograms with the normal distribution curve showed that the data were normally distributed and not violates the parametric assumption for each of the measured dependent variables. Additionally, normality test of data using Shapiro-Wilk test was used, that reflect the data was normally distributed revealed that there was no significant difference with $p$ values of $>0.05$. All these findings allowed the researchers to conduct parametric analysis.
One way within subject MANOVA was conducted to compare between pretreatment and post treatment in (AHD at 0 , AHD at 45, AHD at 60 angles, pain level, and DASH). One way within subject MANOVA revealed that there were significant effects of the measuring periods (the second independent variable) on the tested dependent variables $(\mathrm{F}=36.423, \mathrm{P}=0.000)$.

\section{Acromiohumeral distance at shoulder abduction angles $(0,45,60)$ :}

As presented in table (1) and illustrated in (fig 1), within group's comparison the mean $\pm \mathrm{SD}$ values of AHD at 0 angle in the "pre" and "post treatment" tests were $7.7 \pm 0.52$ and $8.13 \pm 0.63$ respectively. So, the performed multiple comparison revealed that there was significant increase of AHD at 0 angle in post treatment in compare to pretreatment $(\mathrm{P}=0.002)$.

In addition, the mean \pm SD values of AHD at 45 angle in the "pre" and " post treatment" tests were $4.53 \pm 1.02$ and $4.87 \pm 0.96$ respectively as shown in (fig 2). Additionally, the performed multiple compares revealed significant increase of AHD at 45 angle in post treatment in compare to pretreatment $(\mathrm{P}=0.003)$.

Also, the mean \pm SD values of AHD at 60 angle in the "pre" and" post treatment" tests were $6.17 \pm 0.94$ and $6.81 \pm 0.82$ respectively as shown in figure (3). Additionally, the performed multiple comparis revealed significant increase of AHD at 60 angle in post treatment in compare to pretreatment $(\mathrm{P}=0.012)$.

Table 1. One way within subject MANOVAand Descriptive statistics and for AHD at 0, 45, and 60 angles.

\begin{tabular}{|l|l|l|l|}
\hline & $\begin{array}{l}\text { AHD at 0 } \\
\text { angle } \\
\text { Means } \pm \text { SD }\end{array}$ & $\begin{array}{l}\text { AHD at 45 } \\
\text { angle } \\
\text { Means } \pm \text { SD }\end{array}$ & $\begin{array}{l}\text { AHD at 60 } \\
\text { angle } \\
\text { Means } \pm \text { SD }\end{array}$ \\
\hline Pre treatment & $7.7 \pm 0.52$ & $4.53 \pm 1.02$ & $6.17 \pm 0.94$ \\
\hline Post treatment & $8.13 \pm 0.63$ & $4.87 \pm 0.96$ & $6.81 \pm 0.82$ \\
\hline $\begin{array}{l}\text { Multiple comparison tests (Post hoc tests) } \\
\text { pre vs. post treatment) }\end{array}$ \\
\hline P- value & 0.002 & 0.003 & 0.012 \\
\hline
\end{tabular}


SD: standard deviation, $\mathrm{F}$ value: $\mathrm{F}$ statistic value, $\mathrm{P}$ value: probability. *Significant at alpha level $<0.05$.

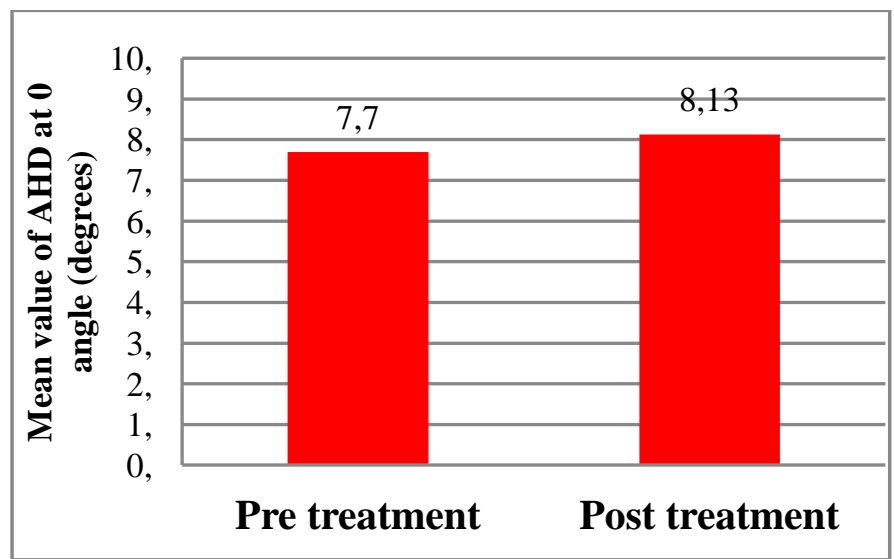

Figure (1) Mean values of AHD at 0 angle pre and post treatments.

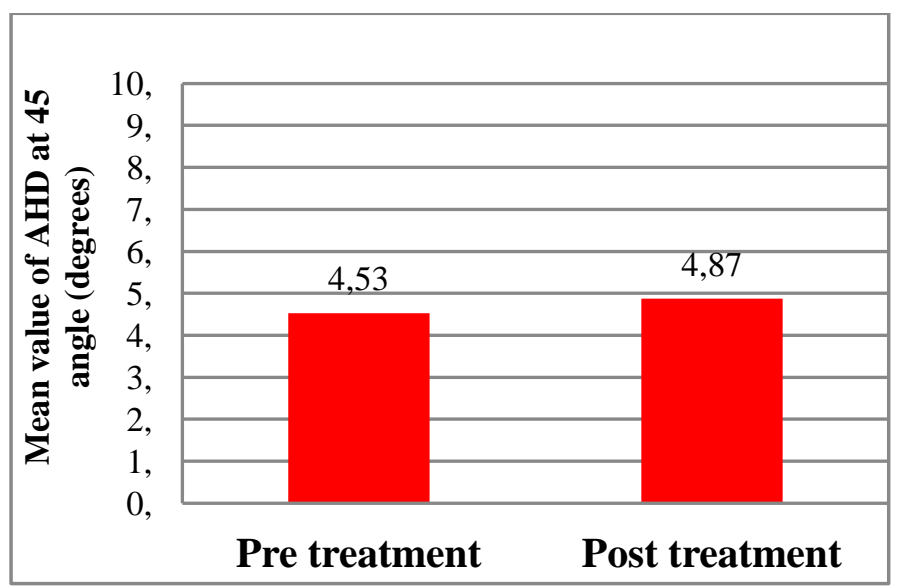

Figure (2) Mean values of AHD at 45 angle pre and post treatments.

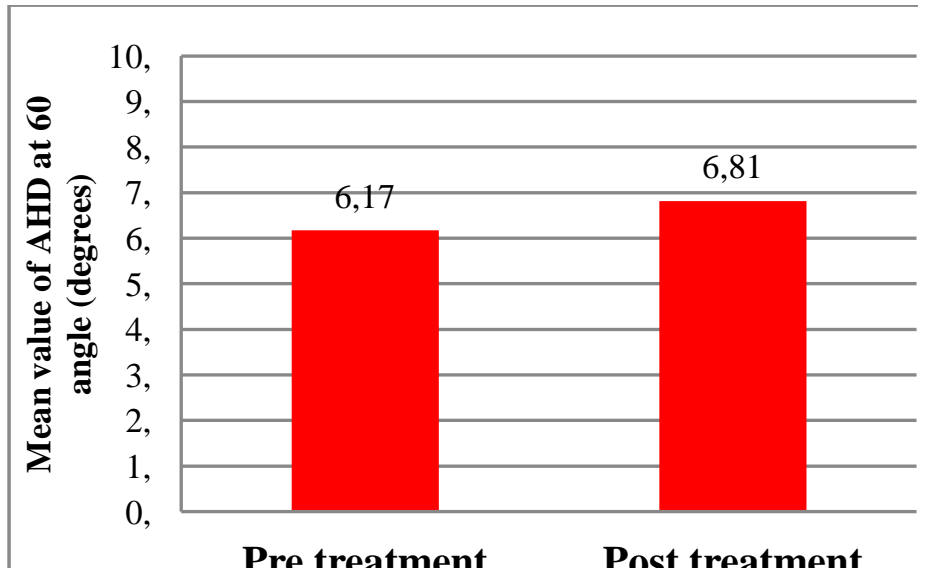

Figure (3) Mean values of AHD at 60 angle pre and post treatments.
[B] Pain and level of upper limb function:

Also, as presented in table (2) and illustrated in figure (4), within group's comparison the mean \pm SD values of pain level in the "pre" and "post treatment" tests were $5.86 \pm 1.12$ and $3.66 \pm 2.12$ respectively. Additionally, the performed multiple comparis revealed significant decrease of pain level in post treatment in compare to pre treatment $(\mathrm{P}=0.000)$. Additionally, the mean $\pm \mathrm{SD}$ values of DASH in the "pre" and "post treatment" tests were $21.07 \pm 12.07$ and $8.43 \pm 7.76$ respectively figure (5). Additionally, the performed multiple comparis revealed significant decrease of DASH in post treatment in compare to pretreatment $(\mathrm{P}=0.000)$.

Table 2. Descriptive statistics and One way within subject MANOVA for pain level and DASH.

\begin{tabular}{|l|l|l|}
\hline & $\begin{array}{l}\text { Pain level } \\
\text { Means } \pm \text { SD }\end{array}$ & $\begin{array}{l}\text { DASH } \\
\text { Means } \pm \text { SD }\end{array}$ \\
\hline Pre treatment & $5.86 \pm 1.12$ & $21.07 \pm 12.07$ \\
\hline Post treatment & $3.66 \pm 2.12$ & $8.43 \pm 7.76$ \\
\hline $\begin{array}{l}\text { Multiple comparison tests (Post hoc tests) (pre vs. post } \\
\text { treatment) }\end{array}$ \\
\hline p-value & $0.000^{*}$ & $0.000^{*}$ \\
\hline
\end{tabular}

SD: standard deviation, $F$ value: $F$ statistic value, $P$ value: probability. ${ }^{*}$ Significant at alpha level $<0.05$.

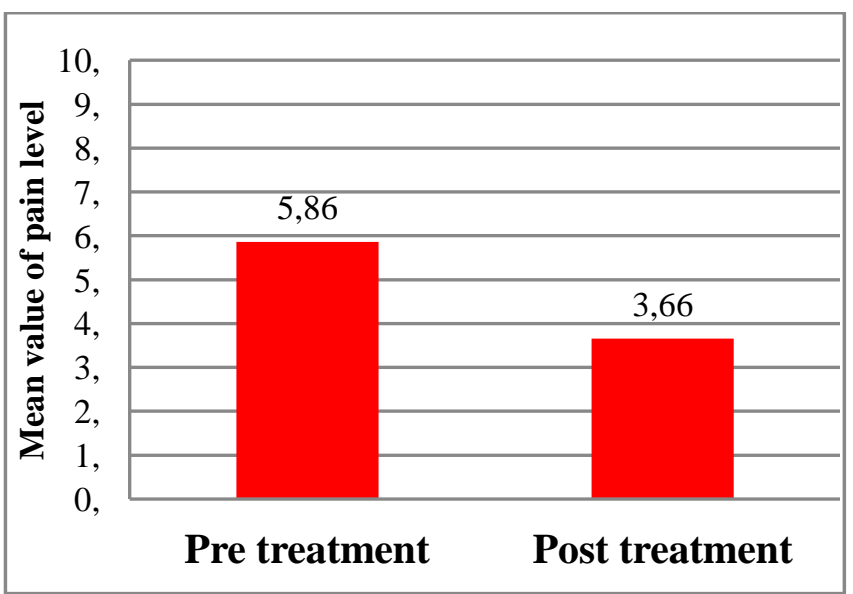

Figure (4): Mean values of pain level pre and post treatments. 


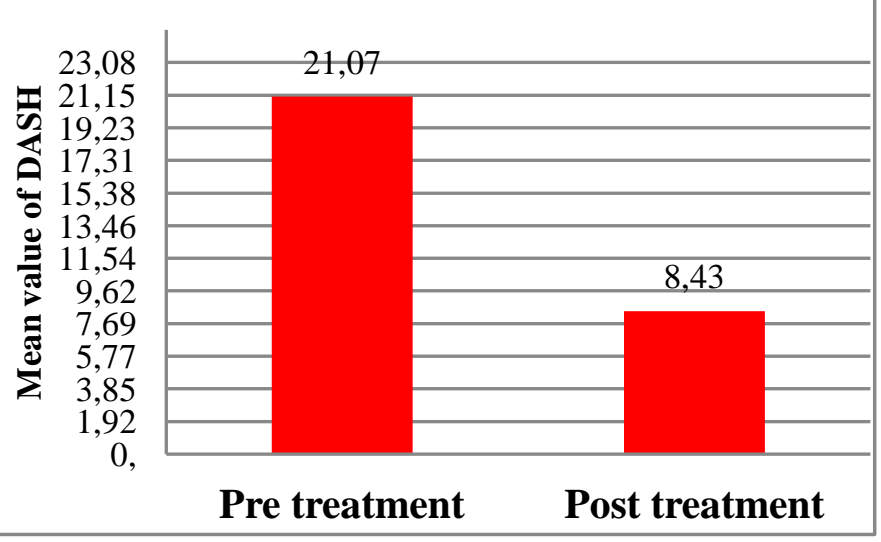

\section{DISSCUSION}

In the present study, we were able to detect a definitive pattern of narrowing of the AHD during shoulder abduction. The obtains data of AHD comply very well with those published previously using different techniques (plain radiographs) by Flatow et all., ${ }^{[14]}$.

In this study, the pattern of narrowing in the AHD measured by the US was confirmed $1.53 \mathrm{~mm}$ (from $7.7 \mathrm{~mm}$ at 0 abduction to 6.17 at 60 abduction) due to superior humeral head migration. Due tech et all.,had a similar pattern of narrowing of AHD using $\mathrm{X}$ - ray, a $1.5 \mathrm{~mm}$ due to superior head migration at 60 shoulder abduction. This gives the ultrasonography the superiority in measuring AHD as the procedure is radiation free ${ }^{[5]}$.

In the present study, the dynamic narrowing pattern of the AHD agrees with the results from a review of the biomechanical mechanism involved in shoulder SIS ${ }^{[15]}$. They suggested that a superior glenohumeral translation in the first portion of abduction due to deltoid muscle action contribute to SIS. A proposed explanation is that the rotator cuff failure due to pain and inhibition need to insufficient humeral head depression to counteract the deltoid action leading to excessive superior migration of the humeral head and reduction of the AHD.

The results of this study found a dynamic narrowing by $3.17 \mathrm{~mm}$ from 0 abduction to 45 abduction in symptomatic patients. An et all, noted a similar pattern of narrowing about $5 \mathrm{~mm}$ when the arm is abducted from 0 to 90 abduction in symptomatic patients with SIS, compared to non-symptomatic patients with narrowing of $2 \mathrm{~mm}$ only ${ }^{[16]}$.
This study showed a definitive and significant $(>0.05)$ improvement in AHD after a 4-week rehabilitation program which confirms the hypothesis that exercise affects the size of AHD. The improvement occurred in all abduction angles (5.58\% at 0 abduction, 7.51 at 45 abduction and 10.37 at 60 abduction). The mean improvement was $(0.43 \mathrm{~mm}$ at 0 abduction, $0.34 \mathrm{~mm}$ at 45 abduction and $0.64 \mathrm{~mm}$ at 60 abduction). These results confirm the role of strengthening exercises program for the rotator cuff muscles to improve the role of the dynamic stabilizers to restrict migration of humeral head superiorly preventing it from encroaching subacromial structures.

The results of this study regarding the improvement of the AHD following a comprehensive rehabilitation program which included posture correction exercises agrees with the results from the study by Carla et all., ${ }^{[17]}$. They investigated the the effect of poor upper quadrant posture on AHD in SIS patients using ultrasonography. They found that there is improvement significantly by $1.2 \mathrm{~mm}$ in AHD after a program concentrated on correction of a slouched posture but no correlation was performed to find out if the improvement of posture and AHD reflected on pain and upper limb function.

Savoie et all., ${ }^{[18]}$ evaluated the effects of a rehabilitation program based on movement training on symptoms, functional limitations and AHD in individuals with SPS. They noted an improvement in AHD in a pattern similar pattern to our study $(0.43 \mathrm{~mm}$ at 0 abduction, $0.34 \mathrm{~mm}$ at 45 abduction and $0.64 \mathrm{~mm}$ at 60 abduction). His study showed mean improvement in AHD after a the rehabilitation program was as following $(0.5 \mathrm{~mm}$ at 0 abduction, $0.9 \mathrm{~mm}$ at 45 abduction and $0.7 \mathrm{~mm}$ at 60 abduction). They noted an improvement of AHD following the exercise program in a manner different than asymptomatic volunteer. The mean AHD of the symptomatic shoulder increased beyond the mean AHD in asymptomatic volunteers. They justified that this larger improvement was due to the fact that the inflamed tendon is already enlarged and occupy a large part of the Subacromial space. 
In the present study, a significant $(<0.05)$ reduction in pain level and improved function was observed after a completion of the rehabilitation program in the SIS group. With a $37.54 \%$ reduction of mean pain level obtained by VAS and with $59.99 \%$ improved on mean DASH score regarding upper limb function.

Although a definitive pattern of AHD narrowing has been confirmed by the results of this study, to our knowledge - surprisingly - no study had examined of the improvement of AHD by US imaging is statistically correlated with pain or upper limb function. Only Mayerhoefer et all., ${ }^{[19]}$ conducted a cross-sectional study aiming to correlate the AHD from MRI and plain radiographs to the level of upper limb function, they concluded that the constant score had a strong positive correlation to the AHD from MRI and plane radiographs.

In light of the results of this study, we can conclude that US measurement of the AHD at the inlet of the subacromial space is a valuable, sensitive and affordable technique that might help who could benefit from the rehabilitation program for SIS patients. further research is needed to confirm this claim.

Therapeutic exercises still prove that's it is a safe cost effective tool in rehabilitations of SIS patients. The present study provides the foundation for the prospective research necessary to fully conclude.

\section{ACHNOWLEGEMENT}

This study wouldn't be completed without the collaborative and team work of my department, special thanks to Dr. Maha mustafa for her constant motivation and advice.

\section{CONCLUSION}

The finding of the present study showed significant changes in all measured variables. The results revealed a significant improvement on AHD in all shoulder angles best noted at angle 60 abduction. Also, a significant reduction in pain level is noted from VAS reports and reduction of the impact of condition on function when the findings of DASH index were analyzed.

\section{REFERENCES}

1. Van der Heijden, G.J., Shoulder disorders: $a$ state-of-the-art review. Baillieres Best Pract Res Clin Rheumatol, 2000. 13(2): p. 287309.

2. Neer, C.S., Anterior Acromioplasty for the Chronic Impingement Syndrome in the Shoulder. 2005.

3. Ludewig, P.M. and J.P. Braman, Shoulder Impingement:

Biomechanical Considerations in Rehabilitation. Manual therapy, 2011. 16(1): p. 33-39.

4. Chen, S.K., et al., Radiographic evaluation of glenohumeral kinematics: a muscle fatigue model. J Shoulder Elbow Surg, 1999. 8(1): p. 49-52.

5. Deutsch, A., et al., Radiologic measurement of superior displacement of the humeral head in the impingement syndrome. Journal of Shoulder and Elbow Surgery, 1996. 5(3): p. 186-193.

6. Seitz, A.L., et al., Mechanisms of rotator cuff tendinopathy: intrinsic, extrinsic, or both? Clinical biomechanics, 2011. 26(1): p. $1-12$.

7. McCreesh, K., et al., Validation of ultrasound measurement of the subacromial space using a novel shoulder phantom model. Ultrasound in medicine \& biology, 2014. 40(7): p. 1729-1733.

8. Ainsworth, R. and J.S. Lewis, Exercise therapy for the conservative management of full thickness tears of the rotator cuff: $a$ systematic review. British journal of sports medicine, 2007. 41(4): p. 200-210.

9. Kuhn, J.E., Exercise in the treatment of rotator cuff impingement: a systematic review and a synthesized evidence-based rehabilitation protocol. Journal of shoulder and elbow surgery, 2009. 18(1): p. 138-160.

10. Pijls, B., et al., Reliability study of the sonographic measurement of the acromiohumeral distance in symptomatic patients. Journal of Clinical Ultrasound, 2010. 38(3): p. 128-134. 
11. Boonstra, A.M., et al., Reliability and validity of the visual analogue scale for disability in patients with chronic musculoskeletal pain. International Journal of Rehabilitation Research, 2008. 31(2): p. 165-169.

12. Hudak, P.L., et al., Development of an upper extremity outcome measure: the DASH (disabilities of the arm, shoulder, and hand). American journal of industrial medicine, 1996. 29(6): p. 602-608.

13. Ellenbecker, T.S. and A. Cools, Rehabilitation of shoulder impingement syndrome and rotator cuff injuries: an evidence-based review. British journal of sports medicine, 2010. 44(5): p. 319-327.

14. Flatow, E.L., et al., Excursion of the rotator cuff under the acromion patterns of subacromial contact. The American Journal of Sports Medicine, 1994. 22(6): p. 779-788.

15. Michener, L.A., P.W. McClure, and A.R. Karduna, Anatomical and biomechanical mechanisms of subacromial impingement syndrome. Clinical biomechanics, 2003. 18(5): p. 369-379.

16. An, K.N., et al., Three-dimensional kinematics of glenohumeral elevation. Journal of Orthopaedic Research, 1991. 9(1): p. 143-149.

17. Kalra, N., et al., Effect of posture on acromiohumeral distance with arm elevation in subjects with and without rotator cuff disease using ultrasonography. journal of orthopaedic \& sports physical therapy, 2010. 40(10): p. 633-640.

18. Mayerhoefer, M.E., et al., Shoulder impingement: relationship of clinical symptoms and imaging criteria. Clinical Journal of Sport Medicine, 2009. 19(2): p. 83-89. 